\title{
A New Strategy for the Surgical Management of RLN Infiltrated by Well-Differentiated Thyroid Carcinoma
}

\author{
Jan Boucek, ${ }^{1}$ Michal Zabrodsky, ${ }^{1}$ Martin Kuchar, ${ }^{1}$ Ondrej Fanta, ${ }^{2}$ \\ Jiri Skrivan, ${ }^{1}$ and Jan Betka ${ }^{1}$ \\ ${ }^{1}$ Department of Otorhinolaryngology, Head and Neck Surgery, First Faculty of Medicine, \\ Charles University in Prague and University Hospital Motol, V Uvalu 84, 15006 Prague, Czech Republic \\ ${ }^{2}$ Department of Anatomy and Biomechanics, Faculty of Physical Education and Sport, Charles University in Prague, \\ Prague, Czech Republic
}

Correspondence should be addressed to Jan Boucek; jboucek99@seznam.cz

Received 7 February 2014; Accepted 26 April 2014; Published 14 May 2014

Academic Editor: Jan Plzák

Copyright (C) 2014 Jan Boucek et al. This is an open access article distributed under the Creative Commons Attribution License, which permits unrestricted use, distribution, and reproduction in any medium, provided the original work is properly cited.

Well-differentiated thyroid carcinoma (WDTC) represents the most common endocrine malignancy. Despite excellent prognoses exceeding $90 \%$ in 10 -year follow-up, there are clinically controversial issues. One of these is extrathyroidal tumour extension invading recurrent laryngeal nerve (RLN). The spread outside of the thyroid parenchyma and invasion to the surrounding structures, classified as always T4a, are the most important negative prognostic factor for the WDTC. Conversely, resection of the RLN leads to vocal cord paralysis with hoarseness, possible swallowing problems, and finally decreased quality of life. We propose a new algorithm for intraoperative management based on the MACIS classification, which would allow swift status evaluation pre/intraoperatively and consider a possibility to preserve the infiltrated RLN without compromising an oncological radicality. In the case of a preoperative vocal cord paralysis (VCP) and confirmation of the invasive carcinoma, a resection of the RLN and the nerve graft reconstruction are indicated. Preoperatively, unaffected vocal cord movement and intraoperatively detected RLN infiltration by the invasive WDTC require an individual assessment of the oncological risk by the proposed algorithm. Preservation of the infiltrated RLN is oncologically acceptable only in specific groups of patients of a younger age with a minor size of primary tumour.

\section{Introduction}

Despite rapidly growing global incidence, well-differentiated thyroid carcinoma (WDTC), arising from follicular cells, papillary and follicular, remains one of the most treatable malignancies [1].

Papillary thyroid carcinoma (PTC) is the most common malignancy of the thyroid gland representing about $83 \%$ of all thyroid cancers. Prognosis is very good with a tenyear survival up to $98 \%[1,2]$. However, there is a group of patients with biologically aggressive WDTC, where a relatively high percentage of persistent disease and recurrences affect long-term morbidity and mortality [3]. Molecular alteration associated with the unfavourable prognosis of the WDTC is genetic and epigenetic alterations of signalling pathways-RAS-RAF-MEK-MAPK-ERK pathway (MAPK pathway) and the PI3K-AKT pathway [1]. From a clinical perspective, the most important negative prognostic factor for the WDTC is a spread outside the thyroid parenchyma and invasion to the surrounding structures, classified in TNM classification as always T4a $[4,5]$. Ten-year survival drops to $45 \%$ compared to patients with the disease restricted to the thyroid gland $[6,7]$.

Among the surrounding structures, the most commonly affected are strap muscles (53\%), recurrent laryngeal nerve (RLN) (47\%), trachea (31\%), esophagus (21\%), and larynx (12\%) [8]. The involvement of the aerodigestive tract (nonRLN invasion) is classified according to Shin et al. [9] in five stages to offer surgical guidelines for therapeutic management. In stage I, when the tumor is located entirely within the thyroid gland capsule with no airway invasion, therapeutic recommendation is a total thyroidectomy. In stage II, when the tumor invades the aerodigestive tract perichondrium or is within a close proximity to the muscle but does not invade 
the cartilage or the muscle deeply, therapeutic recommendation is a complete gross removal by a total thyroidectomy and a partial excision of the cartilage or the muscle. The same therapeutic recommendation is for stage III, when the tumor invades through the airway perichondrium and to the cartilage or to the muscle deeply, but not into the submucosa. In stage IV, the tumor invades through the perichondrium and cartilage or through the muscle and deforms the submucosa but does not penetrate the mucosa and in stage $\mathrm{V}$, there is a gross transmucosal involvement by the carcinoma. A complete resection through the aerodigestive tract is recommended for stages IV and V [9].

Clinically apparent metastatic involvement of the lateral cervical region represents a similarly significant negative prognostic factor as the invasion to the aerodigestive tract, while micrometastases to the central or lateral regions do not affect the prognosis of patients with WDTC [10]. It is shown that a crucial step of the WDTC therapy is a complete surgical removal of the tumor tissue [11, 12] and macroscopically radical resection is the main parameter determining the survival of the patient [13].

\section{Prognostic Significance of the RLN Involvement}

These aspects must be kept in the surgeon's mind considering the management of RLN infiltrated by WDTC. In the context of all mentioned negative prognostic markers, the consideration of the RLN preservation/resection is adequate, only if no other areas, except for the strap muscles, are affected. The surgical radicality remains a subject of debate; the relation to the course of the disease has been established so far only in retrospective studies, not in a large prospective study [7]. Sugitani and Fujimoto demonstrated that patients with the symptomatic papillary microcarcinoma showing preoperative vocal cord paralysis or large node metastasis were likely to die [15]. Also, Ito et al. demonstrated that preoperative vocal cord paralysis may be a sign of a biologically aggressive characteristic; nevertheless, it does not have a significant prognostic value in this study [16].

In particular, the surgical approach in the situation when a WDTC extrathyroid invasion involves only the RLN and a normal vocal cord mobility is preserved remains a controversial issue. Some articles showed no prognostic difference if the affected nerve was either completely resected or when the nerve itself was preserved and the tumor tissue was macroscopically radically removed $[13,17,18]$. However, these studies were retrospective, with a small number of patients and a short follow-up or with nonhomogeneous distribution of extrathyroidal involvement in the compared groups. Nishida et al. did not find any difference in the overall survival, in the number of recurrences, or in the incidence of local, regional, or distant metastases. The study was retrospective and analyzed only with 23 and 27 patients in each group, respectively, and the results were assessed after a relatively short period of follow-up to 6.3 years [18].

Lang et al. retrospectively compared results of 39 and 38 patients, respectively, with the PTC infiltrating the RLN and demonstrated improved short-term and long-term results equally, if the RLN is released from the tumor (by shaving), compared with the radical resection of the infiltrated laryngeal nerve [13]. These two groups were not homogeneous. A significantly higher number of patients with an infiltration of the trachea and other extrathyroidal structures were in the group where the RLN was resected, which considerably impaired oncological results.

"Partial layer resection" constitutes a new, unusual approach when the superficial layer-perineurium-of RLN is resected and the core portion of the nerve is preserved. Functional results were promising, but oncologic results are questionable because of the short follow-up time [19].

Because the intraoperative management of the infiltrated RLN by the WDTC is still controversial issue, we propose a new algorithm based on the MACIS classification [20], which would allow swift evaluation preoperatively and consider the possibility of preserving the infiltrated RLN without compromising oncological radicality.

\section{WDTC Risk Stratification}

For the risk stratification of a particular patient, a number of classifications are used-starting from the international TNM classification (Union Internacional Contra la Cancrum or International Union Against Cancer, American Joint Committee on Cancer-UICC/AJCC) [4], through the AMES classification (age, metastases, extent, and size) [21], the AGES classification (age, grade, extent, and size) [22], and iStage (intraoperative classification according to Ito et al.) [23], finally to the MACIS classification (metastases, age, completeness of excision, invasiveness, and size) [20]. The MACIS classification assesses the completeness or incompleteness of a surgical resection and tumor invasiveness. The MACIS also predicts the development of the WDTC in children and adolescents [24]. A score is obtained by putting values into a formula and a numerical value classifies patient into one of four groups predicting the survival probability (cause-specific survival) in a twenty-year horizon (see Table 1) [5]. The formula is as follows:

(a) for patients less than 40 years old

Total MACIS score $=3.1+(0.3 \times$ tumor size in $\mathrm{cm})$

$$
\begin{aligned}
& +1 \text { (if incomplete excision) } \\
& +1 \text { (if locally invasive) } \\
& +3 \text { (if distant metastases) }
\end{aligned}
$$

(b) for patients older or equal to 40 years

$$
\begin{aligned}
\text { Total MACIS score }= & (0.08 \times \text { age }) \\
& +(0.3 \times \text { tumor size in } \mathrm{cm}) \\
& +1(\text { if incomplete excision }) \\
& +1(\text { if locally invasive }) \\
& +3 \text { (if distant metastases }) .
\end{aligned}
$$




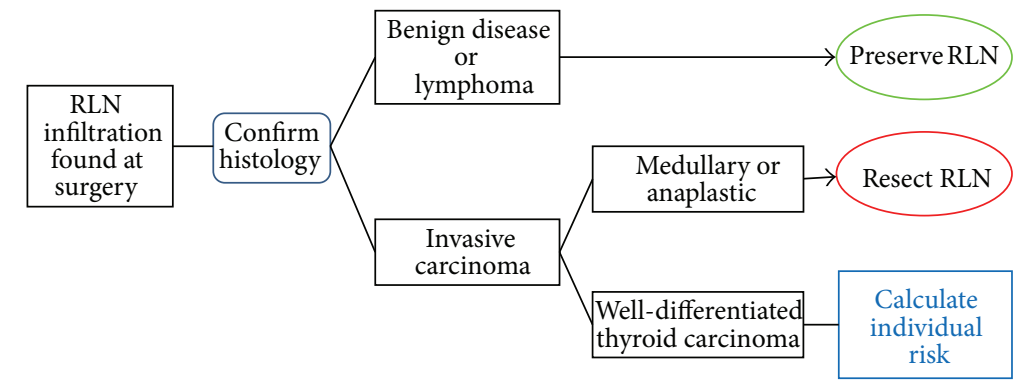

FIGURE 1: Management algorithm of the RLN infiltration found during the surgery (adapted from Richer and Randolph [14]).

TABLE 1: MACIS score and survival.

\begin{tabular}{lc}
\hline Score & Cause-specific survival (\%) \\
\hline$<6$ & 99 \\
$6-6.99$ & 89 \\
$7-7.99$ & 56 \\
$>8$ & 24 \\
\hline
\end{tabular}

\section{Management of the RLN Infiltrated by the WDTC}

From a clinical point of view, the surgeon encounters two situations. The first one is a recurrent laryngeal nerve impairment, which is preoperatively manifested by immobility of the vocal cord (VCP-vocal cord paralysis) on the affected side, often accompanied by a hoarseness and eventually swallowing difficulties. The other possibility is a macroscopic infiltration of the RLN nerve exposed during the surgery, with a normal laryngoscopic examination preoperatively. The current recommendation for the first situation with the preoperative RLN palsy is to verify the biological nature of the disease (by a fine needle aspiration biopsy FNAB or intraoperative frozen section) and preserve the nerve only in a situation of a benign lesion or a lymphoma. If an invasive carcinoma is confirmed, the radical resection including a recurrent laryngeal nerve is indicated [14]. An immediate RLN reconstruction with the greater auricular nerve, during the same surgical procedure as the thyroid cancer extirpation, can provide excellent postoperative phonatory function [25]. It was previously considered that the vocal cord immobility resulting from the preoperative malignant infiltration of the RLN is irreversible $[26,27]$. The analyses of electrophysiological characteristics of RLN seem to be promising in the estimation of the degree of the tumour invasion and distinguishing the cases in which the intraoperative preserving of infiltrated RLN can lead to functionally satisfactory outcome [26, 27].

When the function of RLN is preoperatively not affected and the nerve involvement is found during the operation, some of the current guidelines recommend intraoperative verification of the biological nature of the disease and preservation of the RLN even in the case of an invasive carcinoma, with a maximum effort to remove all macroscopically apparent portions of the tumour around the nerve $[14,27]$. Despite some functional advantages resulting from

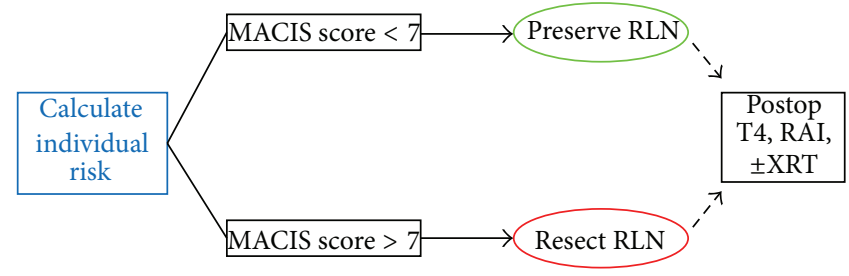

FIGURE 2: A new algorithm of a surgical management of the RLN infiltration based on the MACIS score.

this approach, the oncological safety remains questionable in the case of reducing the emphasis on surgical radicality. If the RLN infiltration by an invasive carcinoma was detected, a surgeon must consider the deterioration of quality of life due to a resection of the recurrent laryngeal nerve on the one hand and compromising of a course of a malignant disease on the other hand. At the same time, it must be emphasized that the consideration to preserve or resect the RLN is legitimate only if there is no invasion of extrathyroidal structures, except for the well resectable strap muscles. However, it is essential to avoid the injury to both recurrent laryngeal nerves, leading to a bilateral palsy, thus dramatically reducing a quality of a patient's life [14, 28].

The algorithm in Figures 1 and 2 is a decision-making strategy which guides the surgeon confronted with infiltration of the RLN. It is based on the analysis of a cause-specific survival (CSS) according to the MACIS classification. We consider a score of less than 6.99 as oncologically acceptable, that is, CSS greater than $89 \%$ (see Figure 3). When there is no metastatic disease and the infliction of the RLN by the WDTC is removable from the nerve without apparent macroscopic residues, the procedure is oncologically safe only in patients under the age of 50, with a tumour size up to $6 \mathrm{~cm}$ (MACIS score $=6.8$ ), under the age of 60 and with the size of the infiltrating tumour up to $3 \mathrm{~cm}$, respectively (MACIS score $=6.7$ ), and under the age of 70, but with only up to $1 \mathrm{~cm}$ of the tumour size, respectively, (MACIS score $=6.9$ ) (see Figure 4). However, in the case of maintaining a continuity of the recurrent laryngeal nerve, the tumour residue remains macroscopically visible, the oncological safety is shifted (see Figure 5), and a preservation of the recurrent laryngeal nerve with grossly apparent residuals is acceptable only in patients at the age of 40 and with tumour size up to $5 \mathrm{~cm}$ (MACIS score $=6.7)$ and in patients under the age of 50 and with 


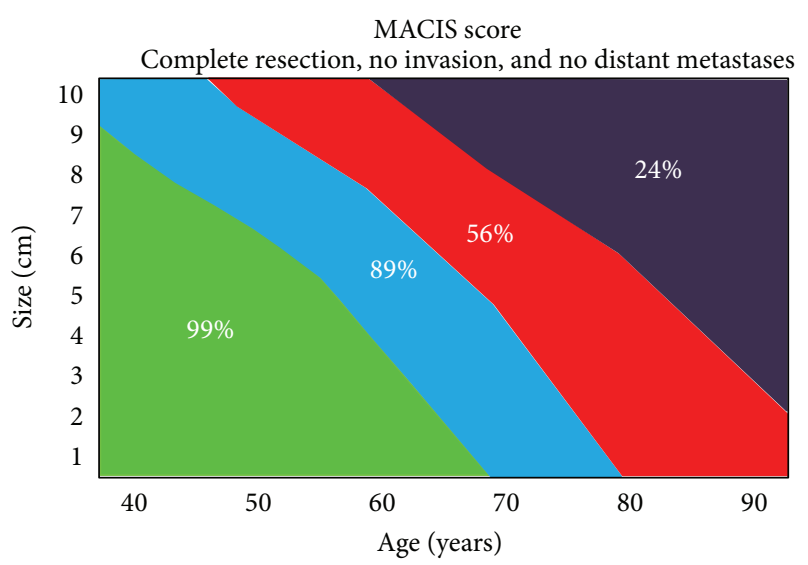

FIGURE 3: Cause-specific survival according to MACIS classification in case of complete resection, no invasion, and no distant metastases.

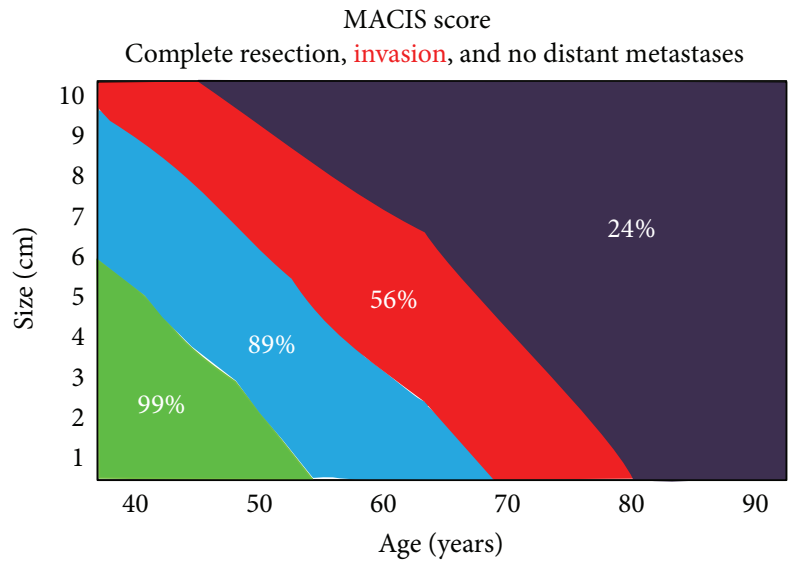

FIGURE 4: Cause-specific survival according to MACIS classification in case of complete resection, invasion, and no distant metastases.

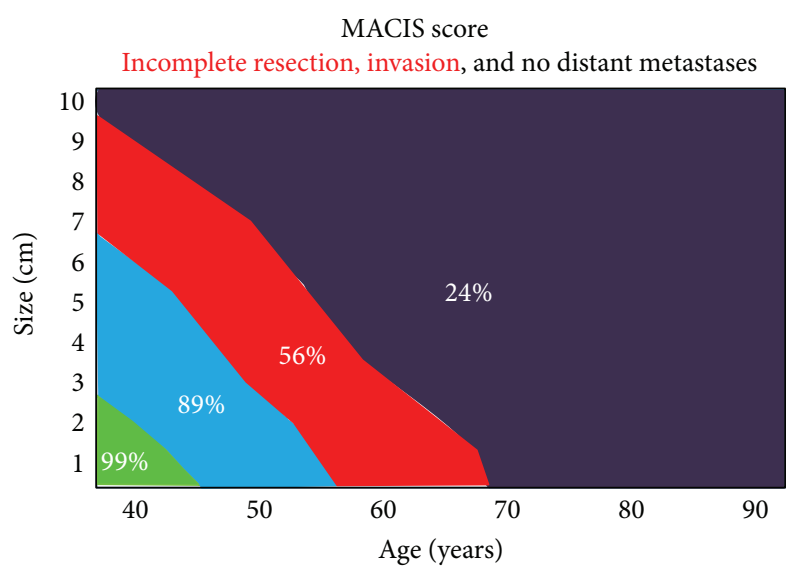

FIGURE 5: Cause-specific survival according to MACIS classification in case of incomplete resection, invasion, and no distant metastases.

the tumour size up to $3 \mathrm{~cm}$ (MACIS score $=6.9$ ), respectively. In elderly patients or larger tumours, the CSS is significantly reduced; that is, the oncological result of the operation would be unacceptable.

\section{Molecular Characteristics of the Aggressive WDTC}

The advances in a molecular and genetic analysis of the WDTC may bring more accurate decision-making strategies in the future. As mentioned above, molecular alterations associated with aggressive behaviour of the WDTC were identified. The gene mutation of serine/threonine kinase BRAF (BRAF V600E), which is a part of the cascade of the MAP kinase group, plays an important role. The pathological activation of the MAPK cascade then causes upregulation of the protooncogenes and downregulation of the tumour suppressor genes in the cell nucleus [1]. At the same time, it adversely affects the iodine absorption of the follicular cells, thus causing the secondary failure of the radioiodine therapy of the PTC. It was demonstrated that the BRAF V600E is detected in up to $95 \%$ of recurrent radioiodine resistant PTC $[29,30]$. A clinical correlation of the BRAF V600E mutation with a worse prognosis for patients with PTC, higher aggressiveness in terms of more frequent regional metastases, an increased incidence of recurrence, and a loss of the radioiodine avidity and thus treatment failure has been demonstrated in many studies and meta-analyses [3, 29, 31-33].

Not much is known about the relationship between biological behaviour of the thyroid tumours and the immune system. The possible negative effects of an antitumor immune response by the regulatory $\mathrm{T}$ cells (Treg) $[34,35]$ are considered here as in other localizations [36-38]. French et al. observed significantly increased number of the Foxp3positive Treg in PTCs showing metastatic disease [35]. A significantly increased number of Treg were also detected by analysis of a FNAB from the lymph nodal PTC metastases, unlike in tumour unaffected nodes [34].

Preoperative knowledge of the presence of these negative prognostic molecular markers would refine the decisionmaking algorithm in high-risk PTC patients (according to conventional risk stratification schemes). The presence of the BRAF V600E mutation would allow a surgeon to act radically and, in the case of an infiltration, sacrifice the RLN nerve. Conversely, in low-risk PTC cases without any evidence of molecular alterations, a conservative approach and a preservation of the nerve are preferable. The possible precision of a molecular analysis of the tumour microenvironment from preoperative cytological samples (FNAB) is now subjects of an intensive research $[31,39,40]$.

\section{Conclusion}

The clinical information about the RLN function and the knowledge of the biological nature of infiltrating tumour is essential for the intraoperative decision-making strategy. In the case of a preoperative VCP and confirmation of the invasive carcinoma, a resection of the RLN and the nerve graft reconstruction are indicated. Preoperatively, unaffected vocal cord movement and intraoperatively detected RLN infiltration by the invasive WDTC require an individual assessment of the oncological risk by the proposed algorithm. Preservation of the infiltrated RLN is oncologically acceptable 
only in specific groups of patients of a younger age with a minor size of primary tumour.

\section{Conflict of Interests}

The authors declare that there is no conflict of interests regarding the publication of this paper.

\section{Acknowledgment}

The work was supported by Grant no. IGA MZ CR NT11542 and by SVV 266513, Prvouk P27, and UNCE 204013.

\section{References}

[1] M. Xing, "Molecular pathogenesis and mechanisms of thyroid cancer," Nature Reviews Cancer, vol. 13, no. 3, pp. 184-199, 2013.

[2] R. M. Tuttle, D. W. Ball, D. Byrd et al., "Thyroid carcinoma," Journal of the National Comprehensive Cancer Network, vol. 8, no. 11, pp. 1228-1274, 2010.

[3] M. Xing, A. S. Alzahrani, K. A. Carson et al., "Association between BRAF V600E mutation and mortality in patients with papillary thyroid cancer," JAMA, vol. 309, no. 14, pp. 1493-1501, 2013.

[4] L. H. Sobin and C. C. Compton, "TNM seventh edition: what's new, what's changed: communication from the International Union Against Cancer and the American Joint Committee on Cancer," Cancer, vol. 116, no. 22, pp. 5336-5339, 2010.

[5] J. C. McCaffrey, "Aerodigestive tract invasion by well-differentiated thyroid carcinoma: diagnosis, management, prognosis, and biology," Laryngoscope, vol. 116, no. 1, pp. 1-11, 2006.

[6] P. E. Andersen, J. Kinsella, T. R. Loree, A. R. Shaha, and J. P. Shah, "Differentiated carcinoma of the thyroid with extrathyroidal extension," American Journal of Surgery, vol. 170, no. 5, pp. 467-470, 1995.

[7] D. L. Price, R. J. Wong, and G. W. Randolph, "Invasive thyroid cancer: management of the trachea and esophagus," Otolaryngologic Clinics of North America, vol. 41, no. 6, pp. 1155$1168,2008$.

[8] T. Tsumori, K. Nakao, and M. Miyata, "Clinicopathologic study of thyroid carcinoma infiltrating the trachea," Cancer, vol. 56, no. 12, pp. 2843-2848, 1985.

[9] D.-H. Shin, E. J. Mark, H. C. S. Hon Chi Suen, and H. C. Grillo, "Pathologic staging of papillary carcinoma of the thyroid with airway invasion based on the anatomic manner of extension to the trachea: a clinicopathologic study based on 22 patients who underwent thyroidectomy and airway resection," Human Pathology, vol. 24, no. 8, pp. 866-870, 1993.

[10] I. J. Nixon and A. R. Shaha, "Management of regional nodes in thyroid cancer," Oral Oncology, vol. 49, no. 7, pp. 671-675, 2013.

[11] J. P. O'Neill and J. P. Shah, "Management of well-differentiated carcinoma with recurrent laryngeal nerve invasion," in Pearls and Pitfalls in Head and Neck Surgery, pp. 30-31, Karger, 2nd edition, 2012.

[12] J. Plzak, J. Astl, G. Psychogios, J. Zenk, P. Laštůvka, and J. Betka, "Current treatment strategies for papillary thyroid microcarcinoma," HNO, vol. 61, no. 4, pp. 300-305, 2013.

[13] B. H. Lang, C. Y. Lo, K. P. Wong, and K. Y. Wan, "Should an involved but functioning recurrent laryngeal nerve be shaved or resected in a locally advanced papillary thyroid carcinoma?" Annals of Surgical Oncology, vol. 20, no. 9, pp. 2951-2957, 2013.
[14] S. L. Richer and G. W. Randolph, "Management of the recurrent laryngeal nerve in thyroid surgery," Operative Techniques in Otolaryngology-Head and Neck Surgery, vol. 20, no. 1, pp. 2934, 2009.

[15] I. Sugitani and Y. Fujimoto, "Symptomatic versus asymptomatic papillary thyroid microcarcinoma: a retrospective analysis of surgical outcome and prognostic factors," Endocrine Journal, vol. 46, no. 1, pp. 209-216, 1999.

[16] Y. Ito, M. Kihara, Y. Takamura, K. Kobayashi, A. Miya, and A. Miyauchi, "Prognosis and prognostic factors of patients with papillary thyroid carcinoma requiring resection of recurrent laryngeal nerve due to carcinoma extension," Endocrine Journal, vol. 59, no. 3, pp. 247-252, 2012.

[17] S. A. Falk, "Management of the recurrent laryngeal nerve in suspected and proven thyroid cancer," Otolaryngology-Head and Neck Surgery, vol. 113, no. 1, pp. 42-48, 1995.

[18] T. Nishida, K. Nakao, M. Hamaji, W. Kamlike, K. Kurozumi, and H. Matsuda, "Preservation of recurrent laryngeal nerve invaded by differentiated thyroid cancer," Annals of Surgery, vol. 226, no. 1, pp. 85-91, 1997.

[19] M. Kihara, A. Miyauchi, T. Yabuta et al., "Outcome of vocal cord function after partial layer resection of the recurrent laryngeal nerve in patients with invasive papillary thyroid carcinoma," Surgery, vol. 155, no. 1, pp. 184-189, 2014.

[20] I. D. Hay, E. J. Bergstralh, J. R. Goellner et al., "Predicting outcome in papillary thyroid carcinoma: development of a reliable prognostic scoring system in a cohort of 1779 patients surgically treated at one institution during 1940 through 1989," Surgery, vol. 114, no. 6, pp. 1050-1058, 1993.

[21] B. Cady, R. Rossi, I. Hay, K. H. Cohn, and N. W. Thompson, "An expanded view of risk-group definition in differentiated thyroid carcinoma," Surgery, vol. 104, no. 6, pp. 947-953, 1988.

[22] I. D. Hay, C. S. Grant, W. F. Taylor, and W. M. McConahey, "Ipsilateral lobectomy versus bilateral lobar resection in papillary thyroid carcinoma: a retrospective analysis of surgical outcome using a novel prognostic scoring system," Surgery, vol. 102, no. 6, pp. 1088-1095, 1987.

[23] Y. Ito, K. Ichihara, H. Masuoka et al., "Establishment of an intraoperative staging system (iStage) by improving UICC TNM classification system for papillary thyroid carcinoma," World Journal of Surgery, vol. 34, no. 11, pp. 2570-2580, 2010.

[24] P. A. Powers, C. A. Dinauer, R. M. Tuttle, and G. L. Francis, "The MACIS score predicts the clinical course of papillary thyroid carcinoma in children and adolescents," Journal of Pediatric Endocrinology and Metabolism, vol. 17, no. 3, pp. 339-343, 2004.

[25] E. Yumoto, T. Sanuki, and Y. Kumai, "Immediate recurrent laryngeal nerve reconstruction and vocal outcome," Laryngoscope, vol. 116, no. 9, pp. 1657-1661, 2006.

[26] D. Kamani, E. A. Darr, and G. W. Randolph, "Electrophysiologic monitoring characteristics of the recurrent laryngeal nerve preoperatively paralyzed or invaded with malignancy," Otolaryngology-Head and Neck Surgery, vol. 149, no. 5, pp. 682-688, 2013.

[27] G. W. Randolph and D. Kamani, "Intraoperative neural monitoring in thyroid cancer surgery," Langenbeck's Archives of Surgery, vol. 399, no. 2, pp. 199-207, 2014.

[28] M. Zabrodsky, J. Bouček, J. Kastner, M. Kuchař, M. Chovanec, and J. Betka, "Immediate revision in patients with bilateral recurrent laryngeal nerve palsy after thyroid and parathyroid surgery. How worthy is it?" Acta Otorhinolaryngologica Italica, vol. 32, no. 4, pp. 222-228, 2012. 
[29] M. Xing, W. H. Westra, R. P. Tufano et al., "BRAF mutation predicts a poorer clinical prognosis for papillary thyroid cancer," Journal of Clinical Endocrinology and Metabolism, vol. 90, no. 12, pp. 6373-6379, 2005.

[30] M. Xing, B. R. Haugen, and M. Schlumberger, "Progress in molecular-based management of differentiated thyroid cancer," The Lancet, vol. 381, no. 9871, pp. 1058-1069, 2013.

[31] J. Y. Joo, J. Y. Park, Y. H. Yoon et al., "Prediction of occult central lymph node metastasis in papillary thyroid carcinoma by preoperative BRAF analysis using fine-needle aspiration biopsy: a prospective study," The Journal of Clinical Endocrinology and Metabolism, vol. 97, no. 11, pp. 3996-4003, 2012.

[32] J. D. Prescott, P. M. Sadow, R. A. Hodin et al., "BRAF V600E status adds incremental value to current risk classification systems in predicting papillary thyroid carcinoma recurrence," Surgery, vol. 152, no. 6, pp. 984-990, 2012.

[33] Y. Ito, H. Yoshida, M. Kihara, K. Kobayashi, A. Miya, and A. Miyauchi, "BRAF mutation analysis in papillary thyroid carcinoma: is it useful for all patients?” World Journal of Surgery, vol. 38, no. 3, pp. 679-687, 2013.

[34] J. D. French, G. R. Kotnis, S. Said et al., "Programmed death$1+\mathrm{T}$ cells and regulatory $\mathrm{T}$ cells are enriched in tumorinvolved lymph nodes and associated with aggressive features in papillary thyroid cancer," The Journal of Clinical Endocrinology and Metabolism, vol. 97, no. 6, pp. 934-943, 2012.

[35] J. D. French, Z. J. Weber, D. L. Fretwell, S. Said, J. P. Klopper, and B. R. Haugen, "Tumor-associated lymphocytes and increased FoxP3+ regulatory $\mathrm{T}$ cell frequency correlate with more aggressive papillary thyroid cancer," Journal of Clinical Endocrinology and Metabolism, vol. 95, no. 5, pp. 2325-2333, 2010.

[36] J. Boucek, T. Mrkvan, M. Chovanec et al., "Regulatory T cells and their prognostic value for patients with squamous cell carcinoma of the head and neck," Journal of Cellular and Molecular Medicine, vol. 14, no. 1-2, pp. 426-433, 2010.

[37] S. Sakaguchi, "Regulatory T cells: key controllers of immunologic self-tolerance," Cell, vol. 101, no. 5, pp. 455-458, 2000.

[38] A. M. Wolf, D. Wolf, M. Steurer, G. Gastl, E. Gunsilius, and B. Grubeck-Loebenstein, "Increase of regulatory T cells in the peripheral blood of cancer patients," Clinical Cancer Research, vol. 9, no. 2, pp. 606-612, 2003.

[39] J. Betka, O. Hovorka, J. Boucek, K. Ulbrich, T. Etrych, and B. Rihova, "Fine needle aspiration biopsy proves increased Tlymphocyte proliferation in tumor and decreased metastatic infiltration after treatment with doxorubicin bound to PHPMA copolymer carrier," Journal of Drug Targeting, vol. 21, no. 7, pp. 648-661, 2013.

[40] A. K. Zimmermann, U. Camenisch, M. P. Rechsteiner, B. BodeLesniewska, and M. Rössle, "Value of immunohistochemistry in the detection of BRAF(V600E) mutations in fine-needle aspiration biopsies of papillary thyroid carcinoma," Cancer Cytopathology, vol. 122, no. 1, pp. 48-58, 2014. 


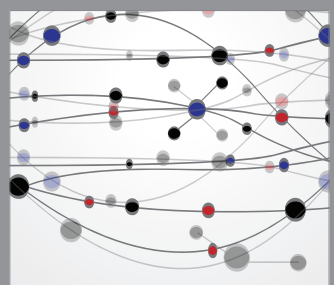

The Scientific World Journal
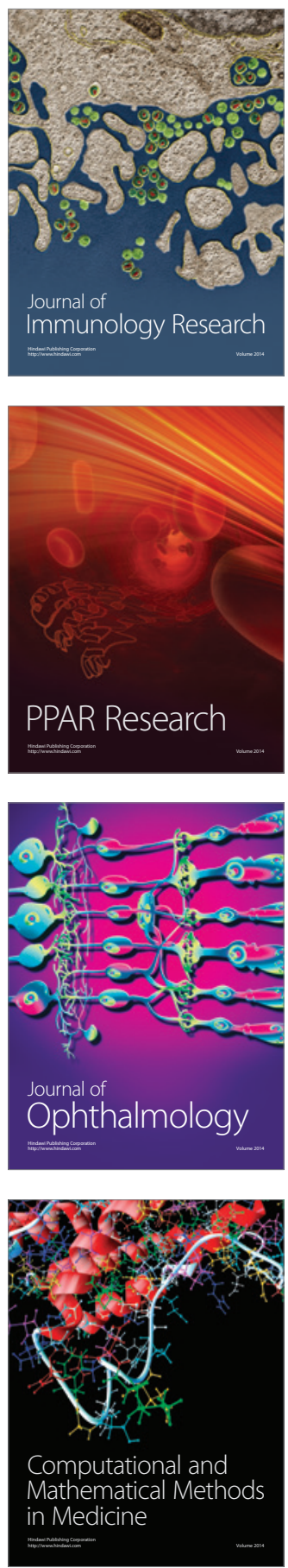

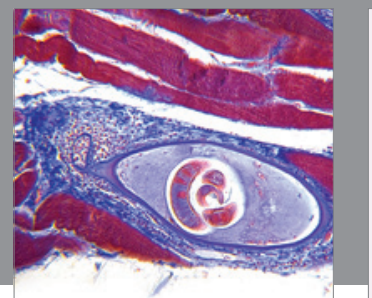

Gastroenterology

Research and Practice
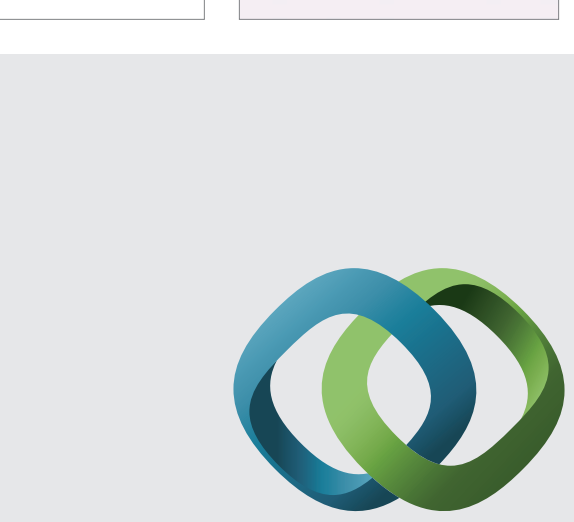

\section{Hindawi}

Submit your manuscripts at

http://www.hindawi.com
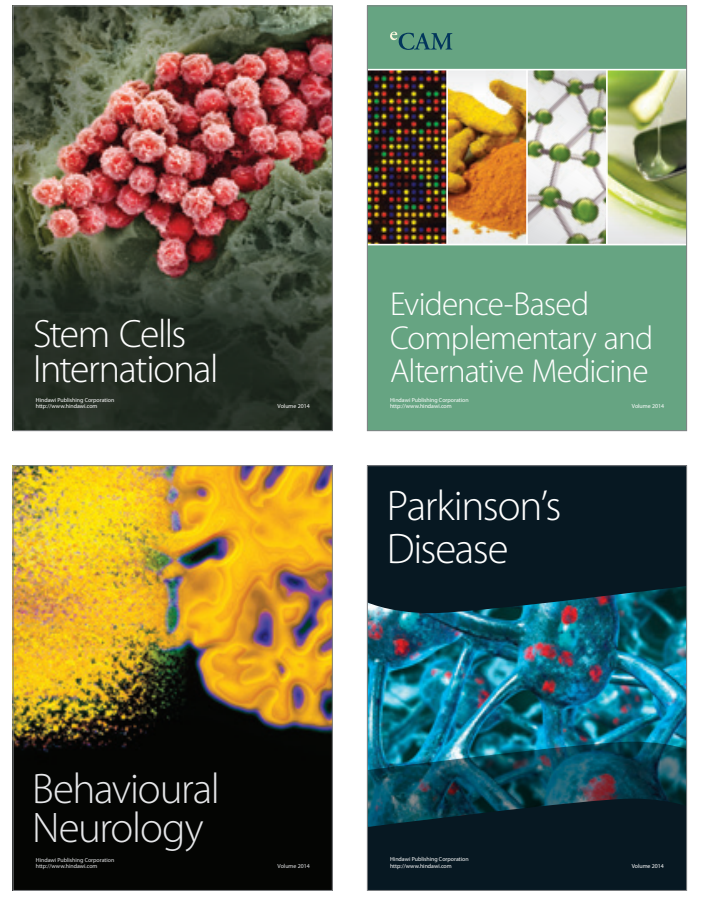
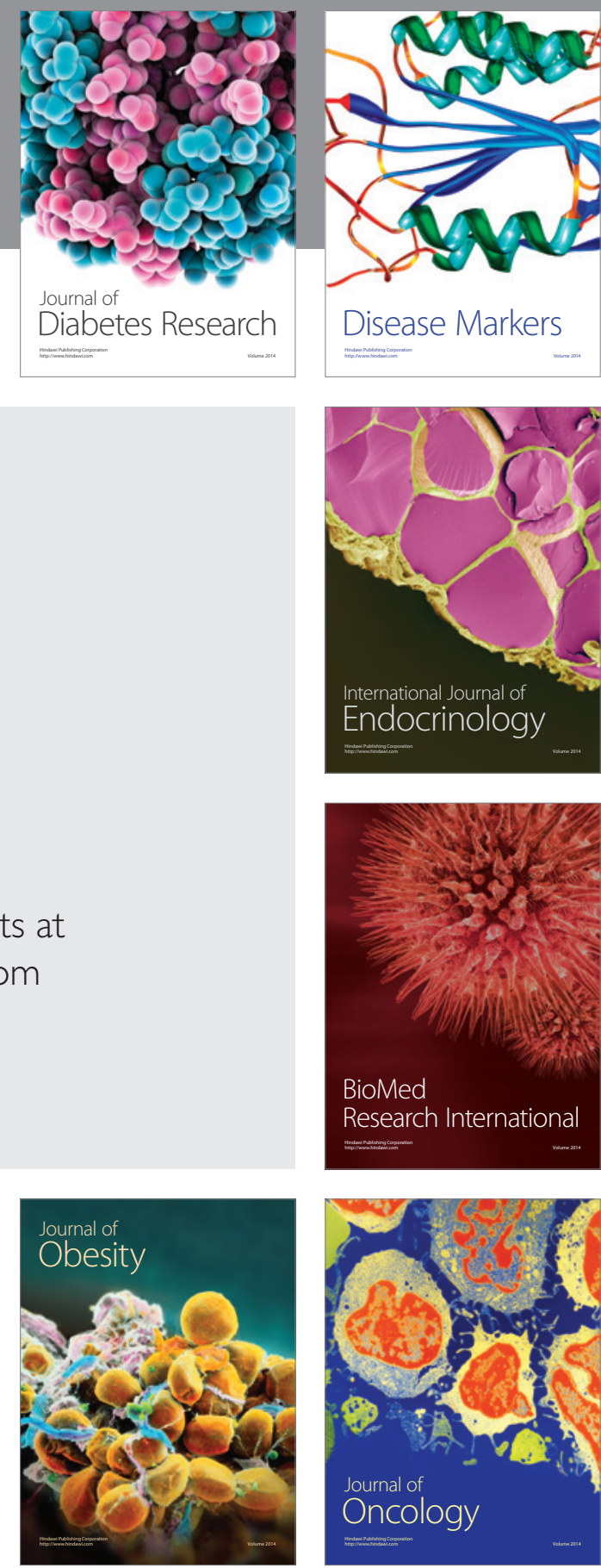

Disease Markers
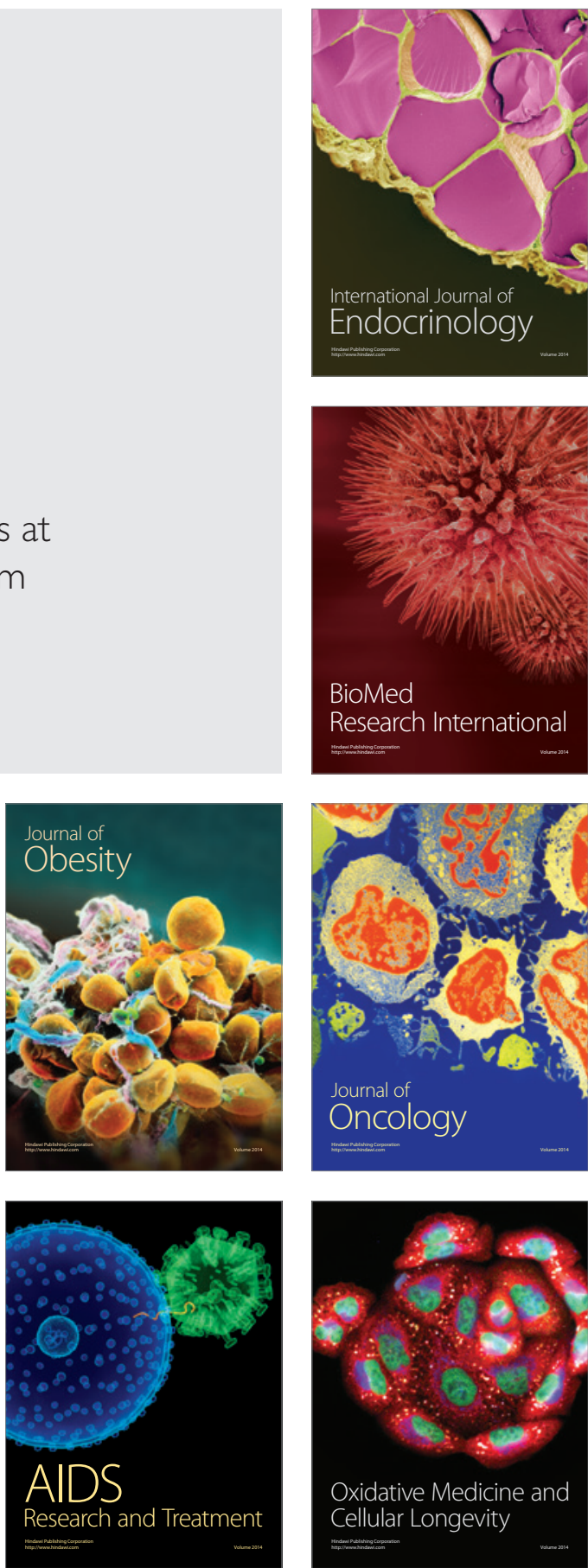